American Journal of Applied Sciences 6 (9): 1669-1674, 2009

ISSN 1546-9239

(C) 2009 Science Publications

\title{
Wave V Detection Using Instantaneous Energy of Auditory Brainstem Response Signal
}

\author{
M.M. Rushaidin, Sh-Hussain Salleh, Tan Tian Swee, J.M. Najeb and Adeela Arooj \\ Center for Biomedical Engineering, Faculty of Biomedical and Health Science Engineering, \\ University Technology Malaysia, 81310 UTM Skudai, Johor, Malaysia
}

\begin{abstract}
Problem statement: ABR machine is a well known machine that has been used on detection of hearing problem especially for babies or children. In ABR, wave V is the most prominent and robust wave that has been used as indicator of hearing loss. However, a fast detection of the wave $\mathrm{V}$ is necessary in order to implement newborn hearing screening. There are various types of signal processing methods introduced by researchers in order to achieve the target. Fast Fourier Transform (FFT) and wavelet transform are well known techniques that have been used in digital signal processing. Approach: In this study, the instantaneous energy of ABR signal had been introduced as a marker to identify the ABR waves. Results: Study showed that the instantaneous energy of auditory brainstem response can be used a marker to identify the ABR waves. Conclusion: This study had proposed a platform for fast hearing screening system.
\end{abstract}

Key words: Auditory brainstem response, hearing screening, instantaneous energy

\section{INTRODUCTION}

Hearing screening: Is an essential test to detect someone's hearing ability. One of the most common neurosensory handicaps in newborns and children is congenital hearing $\operatorname{loss}^{[1,2]}$. Early detection of hearing loss is important to give therapies as early as possible especially to the deaf newborn and children. A serious delay in the development is expected if the therapies were not done to the deaf babies during the first 24 weeks ${ }^{[1,13,14]}$. A good hearing ability is absolutely necessary for a normal speech development. In cases with pronounced hearing loss even no speech ability will be developed at all causing serious communication problems and impaired intellectual and emotional development. The consequences of being deaf-mute are the needs of special schools and care, social isolation and no development of potential skills. Thus there are serious medical and economical consequences for the entire society due to this problem ${ }^{[1]}$.

Data on prevalence of congenital Permanent Childhood Hearing Impairment (PCHI) differs from country to country. This irregularity is partly due to differences in study population, criteria for impairment and the tests that were used in the study. The prevalence of PCHI has been estimated to be 1.1-1.5 for every 1000 live births in Estonia, 1 in 900 in the UK to
1 in 2500 newborns in Atlanta, Georgia. The prevalence of sensorineural hearing loss also varies with race, birth weight and other risk factors. The prevalence of hearing loss in high risk babies is 10-20 times higher than in normal babies. In Malaysia, there is no published data on the actual prevalence of hearing impairment in children. Estimated figures obtained from the Statistics Division, United Nations Economic and Social Commission for Asia and the Pacific (ESCAP) (2002) indicate that Malaysia with a total population of 23.8 million and birth rate of 23.5 per 1000 population has about 559,000 babies born in a year. If the prevalence of hearing loss of 1.5 in every 1000 live births is applied in our population, a rough estimate is that about 840 babies with hearing loss are born each year in this country $^{[2]}$. However, one study case have been done to determine the prevalence of hearing loss among newborns delivered at Hospital University Malaya shows that the prevalence is $0.42 \%(16 / 3,762)^{[10]}$.

Newborn hearing screening program: So far, different Newborn Hearing Screening (NHS) programs to detect the hearing loss as early as possible have been established ${ }^{[3,4]}$. There are two types of hearing test that are commonly used to screen congenital hearing loss; Otoacoustic Emissions (OAEs) and Auditory Brainstem Response (ABR). OAE testing evaluates the integrity of the inner ear (cochlea). In response to noise, vibrations

Corresponding Author: M.M. Rushaidin, Center for Biomedical Engineering,

Faculty of Biomedical and Health Science Engineering, University Technology Malaysia,

81310 UTM Skudai, Johor, Malaysia Tel: +60137027082 Fax: +6075535430

1669 
of the hair cells in a healthy inner ear generate electrical responses, known as otoacoustic emissions. The absence of OAEs indicates that the inner ear is not responding appropriately to sound ${ }^{[3]}$. There are two types of OAE screening techniques, Transient Evoked Otoacoustic Emissions (TEOAEs) and Distortion Product Otoacoustic Emissions (DPOAEs) ${ }^{[3,4]}$.

TEOAEs are generated in response to clicks while DPOAEs are a response to tones. Both stimuli are presented via lightweight ear canal probes. A microphone picks up the signal and multiple responses are averaged to get a reproducible waveform ${ }^{[3]}$. This test can be carried out at the bedside and a pass or fail response is recorded. TEOAE measurements are more commonly used for infant screening whereas DPEOEs are still a subject of research.

The $\mathrm{ABR}$ is an electrophysiological response in the Electroencephalograph (EEG) generated by the brainstem in response to auditory signals such as clicks or tones. The stimulus is delivered via earphones or an inserted ear probe and scalp electrodes are used to pick up the signal. ABR evaluates the integrity of the peripheral auditory system and the auditory nerve pathways up to the brainstem and is able to identify infants who have normal cochlear function but abnormal eighth-nerve function (auditory neuropathy ${ }^{[3]}$. Detection of wave $\mathrm{V}$ in the ABR measurements is the most reliable objective diagnosis and quantification of hearing loss in children ${ }^{[5-8]}$. Due to a poor signal-tonoise ratio, 2000-4000 sweeps have to be averaged to obtain a meaningful, visually noticeable signal at a particular stimulation level ${ }^{[9]}$.

Currently, screening for hearing loss in Malaysia is usually done when babies are brought to the Maternal Child Health Clinics or private clinics for their routine immunization using the Infant Distraction Test or when children fail to attain normal language milestones (personal communication). Recently, newborn hearing screening has been introduced in a few hospitals on a regular basis in the Klang Valley. In other centers, babies with high risk factors such as prolonged NICU stay, craniofacial anomalies or previous history of meningitis are usually referred earlier for a full audiological assessment ${ }^{[2]}$. Typically, screening programs use a 2-stage screening approach, either OAE repeated twice, OAE followed by ABR, or ABR repeated twice, or use a 3-stage screening approach, OAE repeated twice and followed by $\mathrm{ABR}^{[2,3,10-12]}$. NHS programs are commonly conducted as multiple stage procedures. Figure 1 shows the implementation of universal NHS in the state of Saarland, Germany.

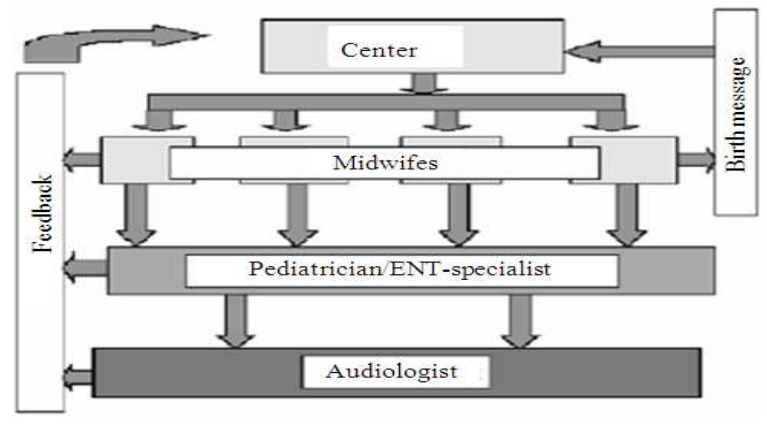

Fig. 1: Organization of 3 stage universal NHS Program (implemented in the state of Saarland, Germany)

ABR recording system: In 1984, an automated electric response audiometry recording system has been developed $^{[7]}$. It uses Nascom 2, an 8 bit microcomputer with a Z80A processor running at $4 \mathrm{MHz}, 32 \mathrm{~KB}$ RAM and 8 input/output ports, 12 bit analog to digital converter, low noise differential amplifier, optically isolated amplifier and DC to DC converter. An electrosensitive printer has been used to record the patient information and the ABR result.

The gain of the amplifier depends on the full-scale voltage range of the $\mathrm{A}-\mathrm{D}$ converter and minimum voltage input requirements ${ }^{[16]}$. Typical gain values for evoked response systems range from 10,000$500,000^{[6,7,16-22]}$. Taking care of the gain of the amplifier is important. It must be low enough to avoid saturation $^{[23]}$.

Frequency bandwidth is important in order to get the correct range for the signal. The frequency bandwidth of the ABR signal, typically range from $20 \mathrm{~Hz}-5 \mathrm{kHz}^{[6,7,9,15,20,24-26]}$. This bandwidth will pass the wave $\mathrm{v}$ and slow SN10 components of the ABR which are important in the estimation of hearing thresholds. There are variety of sampling rate value with variety of resolution bits that has been used to sample the ABR signal; 12.8, 20 and $50 \mathrm{kHz} ; 12$ and 16 bit $^{[6,9,19,20,25-27]}$.

Wave V detection: The detection of responses at threshold levels is not trivial and requires an experienced professional. Different signal detection techniques have been developed and evaluated to improve test efficiency and reliability ${ }^{[18]}$. There are various kind of methods that have been introduced on detection of ABR waves. Strauss et al. ${ }^{[9]}$, introduce an approach to the detection of ABRs using a smart single sweep analysis system. The method used a small number of sweeps which is decomposed by optimized tight frames and evaluated by a kernel based novelty detection machine ${ }^{[9]}$. Woodworth et al. ${ }^{[6]}$, introduce a matched filter that have been derived from an ABR obtained at high stimulus 
level in order to calculate wave $\mathrm{V}$ latency at lower intensity level ${ }^{[6]}$. Wilson and Aghdasi ${ }^{[15]}$, used a Discrete Wavelet Transform (DWT) of ABR signal in order to detect the ABR waves. The wavelets technique is used to decompose a signal into discrete sets of details (high frequencies) and approximations (low frequencies). The different scaled signals are then rebuilt from their resulting wavelet coefficients and analyzed in a method similar to the full signal analysis $^{[24]}$. Delgado and Ozdamar ${ }^{[18]}$, mentioned that results of spectral analysis, spectral filtering and fibertract modeling of ABRs were used to determine the most suitable filters to detect the position of the various peaks. These analyses revealed general trends in ABR composition from one intensity to another and were used to write labeling rules ${ }^{[18]}$. In this study, the instantaneous energy of ABR signal has been introduced as a marker to identify the ABR waves. Instantaneous energy technique has previously been employed in other applications of ECG and heart sound signal processing, such as heart sound segmentation $^{[28]}$.

\section{MATERIALS AND METHODS}

Experiment setup: Figure 2 shows the diagram of the hardware system setup. It consists of (A) gTec USBamp, a biosignal amplifier and data acquisition machine, (B) gPAH, a programmable attenuator, (C) trigger box, (D) headphone, (E) laptop, (F) MP3 player and $(\mathrm{G})$ electrodes.

The gTec USBamp was used to amplify and acquire the ABR signals. The gPAH was used to attenuate the click stimulus produced by MP3 player. MP3 player could be replaced by with a laptop as well.

Different laptops were used in the experiments in order to get smooth clicks. Trigger box was used to produce trigger signal from the stimulus click. The triggered signal were used to segment the ABR signals. The recorded signals were transferred to a laptop by gTec USBamp via USB port. The recorded signal was filtered at bandwidth ranges from $100 \mathrm{~Hz}-3000 \mathrm{kHz}$. The click stimulus rate was setup at 10 clicks sec$^{-1}$ and the signal was sampled at $19.2 \mathrm{kHz}$ with 24 bit resolution. The stimulus intensity levels used in the experiments are 80, 70 and $60 \mathrm{dBnHL}$. The signals were averaged after 2048 click stimulus repetitions. Figure 3 shows the electrodes configuration used in the experiments. The positive electrode (channel 1) was connected to vertex, the negative electrode (reference) was connected to mastoid and ground electrode was connected to forehead.
Matlab R2006a simulink software had been used to capture the raw ABR signals from the gTec USBamp and analyze the signals. However, few configurations need to be carried out using the Matlab model. Figure 4 show the model configuration. The analysis algorithm was written in Matlab M-file format.

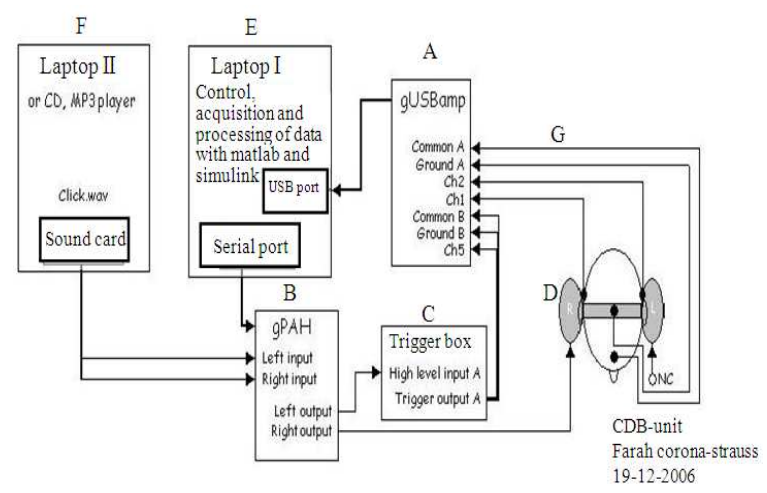

Fig. 2: Diagram of the hardware system setup

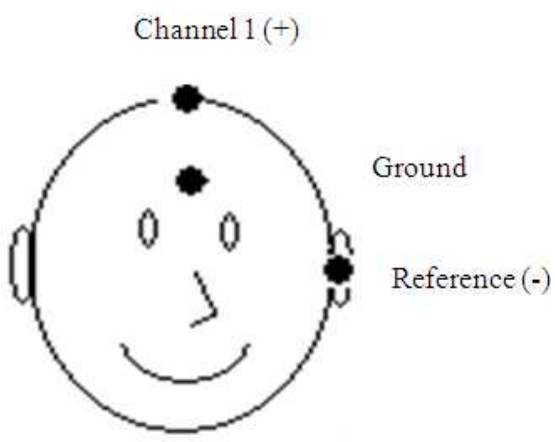

Fig. 3: Electrodes configuration

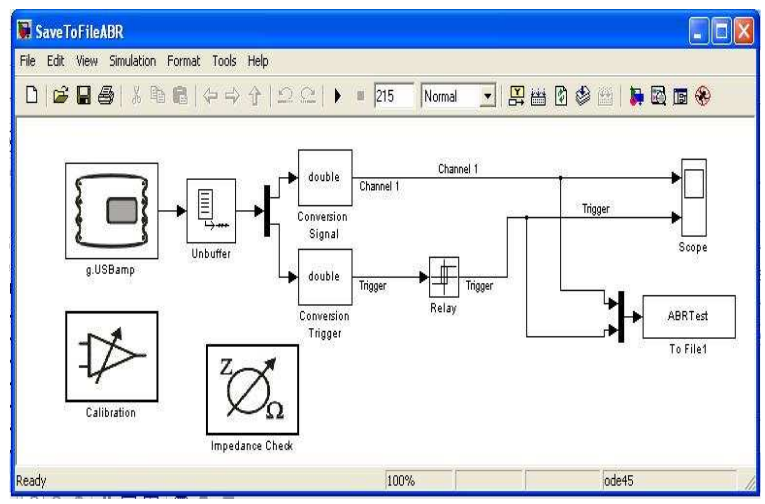

Fig. 4: Matlab model configuration 


\section{RESULTS}

Figure 5 show the presentation of the ABR averaged signal and the instantaneous energy of the averaged signal. Figure 5a shows the result acquired from a hearing loss person and Fig. 5b shows the result acquired from a normal person. Both signals are recorded with the intensity of $80 \mathrm{~dB}$. On the ABR averaging signal graph shows three different averaged signals.

The signals are plotted on three different baselines, 0,1 and $2 \mathrm{uV}$ respectively. The averaged signal on the 0 baseline is used 2000 sweeps, labeled as complete, the averaged signal on the 1 baseline is used first half 1000 sweeps, labeled as 1 st half and the averaged signal on the 2 baseline is used second half 1000 sweeps, labeled as 2nd half. The dotted vertical line marked the latency of wave V. It can be observed that wave V occurred at specific points in the signal of normal person, Fig. 5b, but do not occurred in the signal of hearing loss person, Fig. 5a. Table 1 shows the summary of the graph. The normal subject shows result with the latency $5.208 \mathrm{~m} \mathrm{sec}$ and amplitude range from $0.131-0.26 \mathrm{uV}$. The hearing loss subject shows result with the latency $5.156 \mathrm{~m} \mathrm{sec}$ and amplitude range from -1.1318 to $-0.326 \mathrm{uV}$.

Table 1: Summary of Fig. 5

\begin{tabular}{llllll}
\hline Subject & Intensity $(\mathrm{dB})$ & Latency $(\mathrm{m} \mathrm{sec})$ & Complete $(\mathrm{uV})$ & 1 st half $(\mathrm{uV})$ & 2 ) \\
\hline Normal & 80 & 5.208 & $0.1905-0=0.1905$ & $1.26-1=0.26$ & $2.131-2=0.131$ \\
Hearing loss & 80 & 5.156 & $-0.7266-0=-0.7266$ & $-0.1318-1=-1.1318$ & $1.674-2=-0.326$ \\
\hline
\end{tabular}
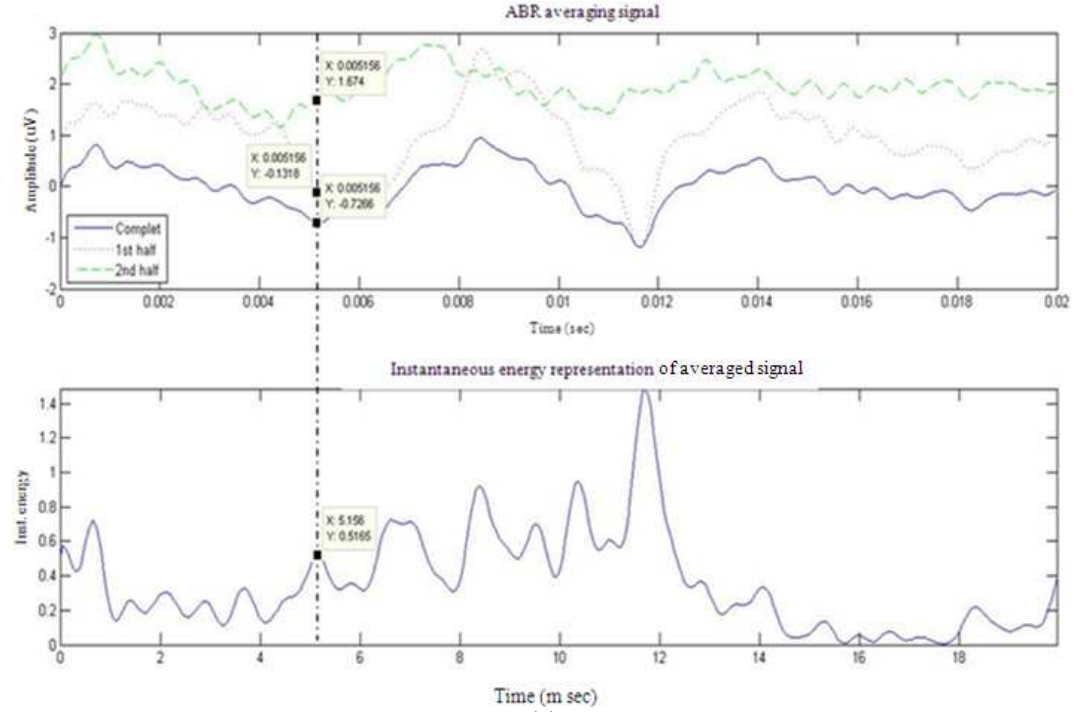

(a)

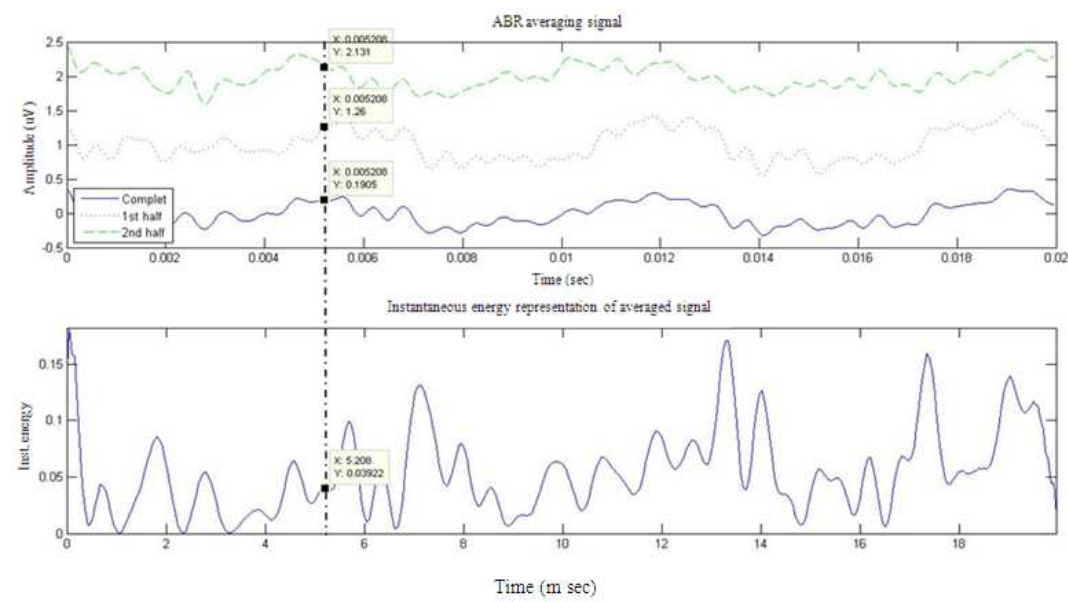

(b)

Fig. 5: Wave V detection using instantaneous energy of ABR signal on (a) hearing loss subject (b) normal subject 1672 


\section{DISCUSSION}

The amplitudes, a, in the Table 1 are obtained by deduction the amplitude appeared on the graph with the baseline. Thus a can be written as

$\mathrm{a}=\mathrm{b}-\mathrm{c}$

Where:

$\mathrm{a}=$ The real amplitude

$\mathrm{b}=$ The graph amplitude

$\mathrm{c}=$ The baseline

The latency of the signals is obtained by considering the intensity of the click and the peak of instantaneous energy of the signal which is near the latency value that has been referring to the latency curve published by Woodworth et al. ${ }^{[6]}$. For the intensity of $80 \mathrm{~dB}$, the latency curve shows that the latency should be within $5 \mathrm{~m} \mathrm{sec}$. In Fig. 5a, the peak of the instantaneous energy is 0.5156 which is near the latency within $5 \mathrm{~m}$ sec. In Fig. $5 \mathrm{~b}$, the peak of the instantaneous energy that near the latency within $5 \mathrm{~ms}$ is 0.03922 .

\section{CONCLUSION}

The new method of the ABR wave's detection such as technique describe in this study is important in order to detect the hearing loss faster especially for infants and children. The results shown in this study that instantaneous energy of ABR signal can be used as marker in order to detect ABR waves. The performance of this method has to be tested.

\section{ACKNOWLEDGEMENT}

This research project is supported by CBE (Center for Biomedical Engineering) at University Technology Malaysia and funded by University Technology Malaysia (UTM), Malaysia under grant "Universal Hearing Screening in Malaysia Based On A Cost Efficient Organization Structure Using An Innovative ABR Technology: The Johor Screening Scheme" Vot 77013.

\section{REFERENCES}

1. Yoshinaga-Itano, C., 1999. Benefits of early intervention for children with hearing loss. Otolaryngol. Clin. North Am., 32: 1089-1102. DOI: $10.1016 / \mathrm{S} 0030-6665(05) 70196-1$
2. Sivalal, S., 2005. Screening for hearing loss in infants. Health Technology Assessment Report. http://www.moh.gov.my/MohPortal/DownloadServ let?id=717\&type $=2$.

3. Helfand, O.M., D.C. Thompson, R. Davis, H. McPhillips, C.J. Homer and T.L. Lieu, 2001. Newborn hearing screening: Systematic evidence review. AHRQ Publication No. 02-S001, Agency for Healthcare Research and Quality, Rockville, $\mathrm{MD}$.

http://www.ncbi.nlm.nih.gov/books/bv.fcgi?rid=hst at3.chapter.1787

4. Delb, W., D. Merkel, K. Pilorget, J. Schmitt and P.K. Plinkert, 2004. Effectiveness of a teoae-based screening program. Can a patient-tracking system effectively be organized using modern information technology and central data management? Eur. Arch. Otorhinolaryngol., 261: 191-196. DOI: 10.1007/s00405-003-0662-3

5. Wicke, J.D., W.R. Goff, J.D. Wallace and T. Allison, 1978. On-line statistical detection of average evoked potentials: Application to Evoked Response Audiometry (ERA). Electroencephalogr. Clin. Neurophysiol., $\quad 44: \quad 328-343$. http://www.ncbi.nlm.nih.gov/pubmed/76539

6. Woodworth, W., S. Reisman and A.B. Fointaine, 1983. The detection of auditory evoked responses using a matched filter. IEEE Trans. Biomed. Eng., 20: 369-376. DOI: 10.1109/TBME.1983.325036

7. Mason, S.M. and W. Adams, 1984. An automated microcomputer based electric response audiometry system for machine scoring of auditory potentials. Clin. Phys. Physiol. Measure., 5: 219-222. DOI: 10.1088/0143-0815/5/3/006

8. Shangkai, G. and M.H. Loew, 1986. An autoregressive model of the BAEP signal for hearing threshold testing. IEEE Trans. Biomed. Eng., $\quad 33$ : $560-565 . \quad$ DOI: 10.1109/TBME.1986.325820

9. Strauss, D.J., W. Delb, P.K. Plinkert and H. Schmidt, 2004. Fast detection of wave $\mathrm{V}$ in ABRs using a smart single sweep analysis system. Proceedings of the 26th Annual International Conference of the IEEE Engineering in Medicine and Biology Society, Sept. 2004, IEEE Xplore Press, pp: 458-461. DOI: 10.1109/IEMBS.2004.1403193

10. Abdullah, A., M.Y.S. Hazim, A. Almyzan, A.G. Jamilah and S. Roslin et al., 2006. Newborn hearing screening: Experience in a Malaysian hospital. Singapore Med. J., 47: 60-64. http://www.sma.org.sg/smj/4701/4701a9.pdf 
11. Clemens, C.J., S.A. Davis and A.R. Bailey, 2000. The false-positive in universal newborn hearing screening. Pediatrics, 106: e7. http://pediatrics.aappublications.org/cgi/content/ful $1 / 106 / 1 / \mathrm{e} 7$

12. Kenedy, C., L. Kimm, R. Thornton and A. Davis, 2000. False positives in universal neonatal screening for permanent childhood hearing impairment. Lancet, 356: 1903-1904. DOI: 10.1016/S0140-6736(00)03267-0

13. Minnesota Department of Health, 2008. Minnesota Early Hearing Detection and Intervention (EHDI) program. Guideline for Organization and Administration of Universal Newborn Hearing Screening Programs in the Well Baby Nursery. http://www.health.state.mn.us/divs/phl/newborn/do cs/nhswbnguidelines.pdf

14. American Speech-Language-Hearing Association (ASHA), 1997. Guidelines for audiologic screening. DOI: 10.1044/policy.GL1997-00199

15. Wilson, W.J. and F. Aghdasi, 1999. Fast fourier transform analysis of the auditory brainstem response: Effects of stimulus intensity and subject age, gender and test ear. AFRICON IEEE., 1: 285-290. DOI: 10.1109/AFRCON.1999.820821

16. American Speech-Language-Hearing Association, 1987. Short latency auditory evoked potentials. ASHA Practice Policy. http://www.asha.org/docs/html/RP198700024.html, doi: 10.1044/policy.RP1987-00024.

17. Neely, S. and Z. Liu, 1998. ABRAV: Auditory Brainstem Response Averager. http://www.boystownhospital.org/Media/Research/ abravtm.pdf

18. Delgado, R.E. and O. Ozdamar, 1994. Automated auditory brainstem response interpretation. IEEE Eng. Med. Biol. Mag., 13: 227-237. DOI: $10.1109 / 51.281682$

19. Dajani, H.R., D. Purcell, W. Wong, H. Kunov and T.W. Picton, 2005. Recording human evoked potentials that follow the pitch contour of a natural vowel. IEEE Trans. Biomed. Eng., 52: 1614-1618. DOI: 10.1109/TBME.2005.851499

20. Boston, J.R., 1981. Spectra of auditory brainstem responses and spontaneous EEG. IEEE Trans. Biomed. Eng., 28: 334-341. DOI: 10.1109/TBME.1981.324801

21. Wilson, W.J. and F. Aghdasi, 2001. The importance of pre-analysis windowing on auditory brainstem response fast Fourier transform analysis. Scand. $\quad$ Audiol., 30: 3-12. http://www.ncbi.nlm.nih.gov/pubmed/11330916.
22. Wilson, W.J., 2004. The relationship between the auditory brain-stem response and its reconstructed waveforms following discrete wavelet transformation. Clin. Neurophysiol., 115: 11291139. DOI: 10.1016/j.clinph.2003.11.019

23. Bell, S.L., D.C. Smith, R. Allen and M.E. Lutman, 2004. Recording the middle latency response of the auditory evoked potential as a measure of depth of anaesthesia. Br. J. Anaesth., 92: 442-445. http://bja.oxfordjournals.org/cgi/content/full/92/3/442

24. Wilson, W.J. and F. Aghdasi, 1999. Discrete wavelet analysis of the auditory brainstem response: Effects of subject age, gender and test ear. AFRICON IEEE., 1: 291-296. DOI: 10.1109/AFRCON.1999.820824

25. Beltran, N. and J.M. Cornejo, 2003. Simultaneous transient evoked otoacoustic emissions and auditory brainstem response for auditory evaluation. Proceedings of the 25th Annual International Conference of the IEEE Engineering in Medicine and Biology Society, Sept. 2003, IEEE Xplore Press, USA., pp: 3222-3224. http://ieeexplore.ieee.org/stamp/stamp.jsp?arnumbe $\mathrm{r}=01280829$

26. Strauss, D.J., W. Delb and P.K. Plinkert, 2004. Objective detection of the central auditory processing disorder: A new machine learning approach. IEEE Trans. Biomed. Eng., 51: 1147-1155. DOI: 10.1109/TBME.2004.827948

27. Firszt, J.B., P.A. Wackym, W. Gaggl, L.S. Burg and R.M. Reeder, 2003. Electrically evoked auditory brain stem responses for lateral and medial placement of the clarion hifocus electrode. Ear Hear., 24: 184-190. http://journals.lww.com/earhearing/Abstract/2003/04000/Electrically_Evoked_ Auditory_Brain_Stem_Responses.9.aspx

28. Malarvili, M.B., I. Kamarulafizam, S. Hussain and D. Helmi, 2003. Heart sound segmentation algorithm based on instantaneous energy of electrocardiogram. Comput. Cardiol., 30: 327-330. DOI: 10.1109/CIC.2003.1291157 